Research Article

\title{
Light Industry Technology of Chiral Perovskite Nanomaterials Construction and Photoelectric Properties
}

\author{
Jie Sun \\ Department of Construction Engineering and Office, Yantai Vocational College, Yantai 264670, Shandong, China \\ Correspondence should be addressed to Jie Sun; 20140912@stu.nun.edu.cn
}

Received 11 June 2021; Revised 20 August 2021; Accepted 25 August 2021; Published 21 September 2021

Academic Editor: Song Jiang

Copyright (c) 2021 Jie Sun. This is an open access article distributed under the Creative Commons Attribution License, which permits unrestricted use, distribution, and reproduction in any medium, provided the original work is properly cited.

\begin{abstract}
Perovskite nanomaterials have become a new research hotspot due to the many novel physical properties of quantum effects and have good application prospects in the field of optoelectronics. Among them, the realization of controllable fabrication of perovskite nanomaterials is the basis of performance studies and applications and is also a difficult point. Reducing production costs to improve manufacturing methods, further improve product controllability, promote advantages, or improve disadvantages will be very important for further application of perovskite nanomaterials. Therefore, this paper studies the construction of titanium ore nanomaterials by designing a novel preparation process and, at the same time, analyzes the related optoelectronic properties of the obtained materials. In this paper, a triangular $\mathrm{PbI}_{2}$ nanosheet having a uniform and controllable shape and size was successfully fabricated by physical vapor deposition through the introduction of a limited space, and related characterization was performed to show uniform controllability and high crystallinity. Using $\mathrm{PbI}_{2}$ nanosheets as the substrate, $\mathrm{MAPbI}_{3}$ nanosheets were further prepared and related characterizations proved that the obtained nanosheets had high crystallinity and excellent optical properties. The experiment proves that the peak position of the fluorescence spectrum obtained by Gaussian fitting is $800 \mathrm{~nm}$ and the half-peak width is about $45 \mathrm{~nm}$. At the same time, $\mathrm{MAPbI}_{3}$ exhibits strong light absorption characteristics when the wavelength is less than $800 \mathrm{~nm}$. This shows that this enclosed space deposition method can improve the uniformity and controllability of $\mathrm{PbI}_{2}$ and $\mathrm{MAPbI}_{3}$ nanosheet fabrication, which provides the basis for further research on $\mathrm{MAPbI}_{3}$ nanomaterials and references to the controllable growth of other nanomaterials.
\end{abstract}

\section{Introduction}

As a direct band gap semiconductor, perovskite has many advantages, such as designable structure, continuously adjustable band gap, high light absorption and emission efficiency, high carrier mobility, and low preparation cost. It has become a star material that has attracted much attention. When the perovskite is further made into nanomaterials, due to the quantum effect, it will exhibit many novel physical and chemical properties, further expanding its application range. Perovskites also have excellent electrical properties, high hole mobility, and carrier diffusion lengths up to the millimeter level. When perovskites form nanomaterials, they have newer properties due to quantum effects. Because of these outstanding optical and electrical properties, perovskites, especially perovskite nanomaterials, have been extensively studied.
The study of the structure and optoelectronic properties of perovskite nanomaterials abroad is much faster than in China, the discovery of perovskite nanomaterials is also faster than in China, and the construction technology of nanomaterials is rapidly developing and updating. The construction and optoelectronic properties of perovskite nanomaterials have been greatly improved and developed. It is believed that the application of perovskite nanomaterials in light industry will become more and more extensive in the near future. Ha et al. synthesized a series of tantalate compounds with Dion-Jacobson layered perovskite structure by solid-phase method. These compounds can photocatalytically decompose water to produce hydrogen and oxygen under the irradiation of ultraviolet light [1]. Halford studied the Ruddlesden-Popper layered perovskite structure compound and found that it can effectively degrade 
rhodamine B solution under ultraviolet light irradiation and have a wide range of applications in photocatalytic sterilization, water purification treatment, and conversion of solar energy to hydrogen energy [2]. Riba et al. prepared a series of perovskite nanoparticles with different compositions and realized the continuous adjustment of its fluorescence spectrum from $410 \mathrm{~nm}$ to $710 \mathrm{~nm}$ in the visible light region [3].

The application of perovskite nanomaterials in light industry began in western countries. Research on perovskite nanomaterials in our country started late compared to western countries, and the development speed is relatively slow. With the continuous development of science and technology and the maturity of nanomaterials, studying the construction and photoelectric properties of perovskite nanomaterials can greatly promote the development of our country's light industry. Shang et al. successfully prepared a new and highly active solid solution catalyst, which can effectively degrade the organic pollutant acetaldehyde in response to visible light [4]. Han et al. synthesized perovskite nanosheet structures of different thicknesses through the solution method and tested the PL of samples with different thicknesses. The results found that as the nanosheets increased from a single layer to 10 layers, the PL peak position shifted significantly from $724 \mathrm{~nm}$ [5]. Kong et al. observed ionic conductivity higher than electron conductivity in perovskite nanomaterials, indicating the importance of ion transport in perovskite [6].

This paper improves the perovskite vapor phase synthesis method, reduces the cost of preparing the vapor phase method, and obtains a uniform and controllable perovskite nanomaterial. Although we have achieved some results from the preparation of the material, there are still aspects that require further development. Although the uniform and controllable $\mathrm{PbI}_{2}$ and $\mathrm{MAPbI}_{3}$ nanosheets have been obtained by the confined space method, there is a lack of indepth research on the method and the materials obtained. The dynamic instability in the growth process of nanomaterials is universal. This experiment only improves the preparation of $\mathrm{PbI}_{2}$ nanosheets to overcome this phenomenon, but the same problems existing in the preparation of other nanomaterials have not been explored in depth.

\section{Construction and Photoelectric Properties of Chiral Perovskite Nanomaterials}

\subsection{Optical and Electrical Properties of Perovskite}

2.1.1. Optical Properties. Perovskite is a direct band gap semiconductor with a high absorption coefficient, so it has excellent optical properties. In addition, the perovskite band gap position is appropriate, between 1.4 and $3.0 \mathrm{eV}$, and the light absorption and emission can just cover the entire visible light region, which has broad application prospects. The optical performance adjustment can be achieved between the same type of perovskite through ion exchange, and the same can be achieved between different types of perovskite. When perovskite forms nanomaterials, due to the existence of quantum effects, its optical properties will also change with the thickness of perovskite nanomaterials [7, 8]. From the single layer to the bulk phase, the bandgap offset can reach $100 \mathrm{meV}$, which shows that the thickness of the perovskite nanosheets has a great influence on the optical properties.

2.1.2. Electrical Properties. In addition to excellent optical properties, perovskite has good electrical properties, and perovskite has high ionic conductivity. The conductivity of perovskite is not only related to temperature and $\mathrm{I}_{2}$ partial pressure, but also related to light $[9,10]$. As the light intensity increases, the ionic and electronic conductivities continue to increase. Second, perovskites also have a longer carrier diffusion distance and lower defect concentration. The optical and electrical properties of $\mathrm{MAPbX}_{3}$ single crystal have a carrier diffusion distance exceeding $10 \mu \mathrm{m}$, and the crystallinity is the same as the highest crystalline quality of single crystal silicon.

\subsubsection{Application of Perovskite Nanomaterials in the Field of} Optoelectronics. Among them, perovskite nanomaterials such as nanowires and nanosheets generally have very high crystallinity and better performance. At the same time, its small size makes it particularly suitable for applications in the field of micronano optoelectronic devices. The principle of photodetectors is based on the photoelectric effect of semiconductor materials. The photoelectric effect can be divided into the photoconductive effect and the photovoltaic effect $[11,12]$. A good photodetector needs to meet the conditions of high spectral selectivity, high sensitivity, fast response speed, high signal-to-noise ratio, and good cycle stability.

\subsection{Preparation of Perovskite Nanomaterials. Perovskite has} the advantages of simple method and low cost in preparation, which is also an important reason for its large-scale application in the field of optoelectronics. The raw materials for perovskite synthesis are usually $\mathrm{AX}$ and $\mathrm{BX}_{2}$. The onestep method refers to the direct reaction of the two to obtain perovskite nanomaterials. The two-step method refers to the synthesis of $\mathrm{BX}_{2}$ precursor nanomaterials and then further conversion with AX into perovskite [13, 14]. No matter which method is used to prepare the corresponding nanorods and nanosheets of perovskite nanomaterials, machine learning models need to be used to detect their optoelectronic properties.

2.2.1. Decision Tree. In the process of constructing the decision tree model, the most important thing is the feature selection of the node. Through the feature selection, the standard for constructing the split judgment is reached and the tree is further split. The Gini index is the main method to select the optimal feature in the decision tree model [15]. When the decision tree model is used for classification, if there are $i$ categories and $p_{i}$ is the probability that the sampling point belongs to the $i$ category, then the Gini index formula is 


$$
\operatorname{Gini}(p)=\sum_{i=1}^{I} p_{i}\left(1-p_{i}\right)=1-\sum_{i=1}^{I} p_{i}^{2}
$$

When the decision tree performs two classifications, assuming that the probability of the sample point being classified into the first class is $q$, then the Gini index of the probability distribution is

$$
\operatorname{Gini}(q)=2 q(1-q)
$$

For a given sample set $M$, its Gini index is

$$
\operatorname{Gini}(M)=1-\sum_{i=1}^{I}\left(\frac{\left|C_{i}\right|}{|M|}\right)^{2}
$$

Here, $C_{i}$ is the subset of samples in $M$ that is the class $i$ and $M$ is the number of classes.

2.2.2. Logistic Regression. Taking $X$ as a continuous random variable, which obeys the logistic distribution, its distribution function and density function are

$$
\begin{aligned}
F(x) & =P(X \leq x) \\
& =\frac{1}{1+e^{-(x-\mu) / \gamma}}, \\
f(x) & =F^{\prime}(x) \\
& =\frac{e^{-(x-\mu) / \gamma}}{\gamma\left(1+e^{-(x-\mu) / \gamma}\right)^{2}} .
\end{aligned}
$$

Binomial logistic regression in logistic regression can be used as a classification, with conditional probability $P(Y \mid X)$ as a model function. In the model, the value of random variable $X$ can be any real number, and the corresponding $Y$ value is 1 or $0[16,17]$. Its conditional probability distribution is

$$
\begin{aligned}
P(Y & =1 \mid x) \\
& =\frac{\exp (\omega x+b)}{1+\exp (\omega x+b)}, \\
P(Y & =0 \mid x) \\
& =\frac{1}{1+\exp (\omega x+b)} .
\end{aligned}
$$

Here, $x \in R^{n}$ is the input, $Y \in\{0,1\}$ is the output, $\omega \in R^{n}$ and $n \in R^{n}$ are the parameters. We call $\omega$ the weight vector and $b$ the bias. Let

$$
\begin{aligned}
& P(Y=1 \mid x)=\pi(x), \\
& P(Y=0 \mid x)=1-\pi(x) .
\end{aligned}
$$

The likelihood function is

$$
\prod_{i=1}^{N}\left[\pi\left(x_{i}\right)\right]^{y_{i}}\left[1-\pi\left(x_{i}\right)\right]^{1-y_{i}} .
$$

The log likelihood function is

$$
L(w)=\sum_{i=1}^{N}\left[y_{i}(w x)-\log (1+\exp (w x))\right] .
$$

Find the maximum value of $L(w)$ to get the estimated value of $w$. Assuming that the maximum likelihood estimate of $w$ is $\widehat{w}$, the regression model obtained by training is

$$
P(Y \mid X)=\frac{\exp (\widehat{\omega} x)}{1+\exp (\widehat{\omega} x)} .
$$

2.2.3. Support Vector Regression Algorithm. (1) Linear Support Vector Regression Machine. Suppose the sample data set is represented as $\varphi: R^{n} \longrightarrow H\left\{x_{k}, y_{k}\right\}_{k=1}^{N}$, where $N$ is the number of samples, the input data $x_{k} \in R^{n}$ is $n$ dimensional, and the output data $y_{k} \in R^{n}$ corresponds to the input data $x_{k}$ $[18,19]$. Suppose the linear regression function is

$$
f(x)=w^{T} x+b,
$$

where $w \in R^{n}, b \in R$, and $w$ are normal vectors. The loss function is used as follows:

$$
|y-f(x)|_{\varepsilon}= \begin{cases}0, & \text { if }|y-f(x)| \leq \varepsilon, \\ |y-f(x)|-\varepsilon, & \text { otherwise. }\end{cases}
$$

Among them, $\varepsilon$ is the preset tolerable loss function, and the distance between the two dotted lines is $2 /\|w\|$. The optimization goal of the algorithm is to maximize the distance, that is, minimize the reciprocal of the logarithmic distance $[20,21]$. Finally, the process of finding the most suitable regression function is transformed into

$$
\min \frac{1}{2}\|w\|^{2}
$$

The penalty parameter $C$ and the relaxation factor $\xi_{i}, \xi_{i}^{*}$ are introduced, and the optimization problem corresponding to the regression estimation is transformed into

$$
\min _{w, b} P=\min \frac{1}{2}\|w\|^{2}+C \sum_{i=1}^{N}\left(\xi_{i}+\xi_{i}^{*}\right)
$$

The constraints that should be met are

$$
\begin{cases}y_{i}-w^{T} x_{i}-b \leq \varepsilon+\xi_{i}, & i=1, \ldots, N, \\ w^{T} x_{i}+b-y_{i} \leq \varepsilon+\xi_{i}, & i=1, \ldots, N \\ \xi_{i}, \xi_{i}^{*} \geq 0, & i=1, \ldots, N .\end{cases}
$$

Lagrangian dual functions are usually introduced to solve the above convex quadratic programming problem [22]. The Lagrangian dual function is constructed as follows: 


$$
\begin{aligned}
L\left(w, b, \xi_{i}, \xi_{i}^{*}, a, a^{*}, \eta, \eta^{*}\right)= & \frac{1}{2}\|w\|^{2}+C \sum_{i=1}^{N}\left(\xi_{i}+\xi_{i}^{*}\right) \\
& -\sum_{i=1}^{N} a_{i}\left(\varepsilon+\xi_{i}-y_{i}+w^{T} x_{i}+b\right) \\
& -C \sum_{i=1}^{N}\left(\eta_{i} \xi_{i}+\eta_{i}{ }^{*} \xi_{i}^{*}\right) .
\end{aligned}
$$

The Lagrangian multiplier is introduced, which turns the problem into a solution:

$$
\max _{a, a^{*}, \eta, \eta^{*}} \min _{w, b, \xi, \xi^{*}} L\left(w, b, \xi, \xi^{*}, a, a^{*}, \eta, \eta^{*}\right) .
$$

Taking the Lagrangian dual function to differentiate $w, b, \xi$, and $\xi^{*}$ equal to zero, the dual problem can be transformed into a convex quadratic programming problem:

$$
\begin{aligned}
\max _{a, a^{*}} J_{D}\left(a, a^{*}\right)= & -\frac{1}{2} \sum_{k, i=1}^{N}\left(a-a^{*}\right)\left(a_{k}-a_{k}^{*}\right) x_{i}^{T} x_{k} \\
& -\varepsilon \sum_{i=1}^{N}\left(a_{i}+a_{i}^{*}\right)+\sum_{i=1}^{N} y_{i}\left(a_{i}+a_{i}^{*}\right) .
\end{aligned}
$$

The conditions are

$$
\sum_{i=1}^{N}\left(a_{i}-a_{i}^{*}\right)=0, \quad a_{i} a_{i}^{*} \in[0, c] .
$$

The final target fitting regression function is expressed as

$$
f(x)=\sum_{i=1}^{N}\left(a_{i}-a_{i}^{*}\right) x_{i}^{T} x+b .
$$

Through deduction, it can be seen that the number of support vectors in SVM is limited. The input samples $x_{i}$ corresponding to those $a_{i}$ are not zero. Only input vectors that meet this condition can contribute to the SVM model. This is the sparsity of the SVM solution $[23,24]$.

(2) Nonlinear Support Vector Regression Machine. Polynomial kernel functions have better generalization power and belong to global kernel functions, but the price paid is an unsuitable effect on nonlinear problems. The nonlinear SVM problem can be expressed as solving the following problem:

$$
\begin{aligned}
\max _{a, a^{*}} J_{D}\left(a, a^{*}\right)= & -\frac{1}{2} \sum_{k, j=1}^{N}\left(a_{i}-a_{i}^{*}\right)\left(a_{k}-a_{k}^{*}\right) K\left(x_{i}, x_{k}\right) \\
& -\varepsilon \sum_{i=1}^{N}\left(a_{i}-a_{i}^{*}\right)+\sum_{i=1}^{N} y_{i}\left(a_{i}-a_{i}^{*}\right) .
\end{aligned}
$$

The conditions are

$$
\sum_{i=1}^{N}\left(a_{i}-a_{i}^{*}\right)=0, \quad a_{i} a_{i}^{*} \in[0, c] .
$$

The final target fitting regression function is expressed as

$$
f(x)=\sum_{i=1}^{N}\left(a_{i}-a_{i}^{*}\right) K\left(x_{i}, x\right)+b .
$$

When there is a lack of prior knowledge in the relevant fields of sample data, the radial basis kernel function is generally selected as the kernel function, which can better balance the fitting effect and generalization ability than other kernel functions [25]. The expression of the radial basis kernel function is as follows:

$$
K\left(x^{\prime}, x\right)=\exp \left(\frac{-\left\|x-x^{\prime}\right\|^{2}}{\sigma^{2}}\right),
$$

where $\sigma$ is a parameter that characterizes the width of the core.

\section{Construction of Perovskite Nanomaterials and Experimental Design of Photoelectric Properties}

\subsection{Purpose}

(1) Research on the construction of perovskite nanomaterials

(2) Study of the optoelectronic properties of perovskite nanomaterials

3.2. Preparation of $\mathrm{PbI}_{2}$ Nanosheets. $\mathrm{PbI}_{2}$ is an important precursor material in the process of preparing perovskite nanomaterials by the two-step method, and $\mathrm{PbI}_{2}$ itself is a layered structure, and it is easy to prepare a two-dimensional nanosheet structure through regulation. The two-dimensional $\mathrm{PbI}_{2}$ preparation is prepared by vapor deposition method. As shown in Figure 1, this preparation method can meet the requirements of productivity and crystallinity at the same time. Based on the high-quality $\mathrm{PbI}_{2}$ nanosheets obtained, the vapor deposition method is further used to transform it into $\mathrm{MAPbI}_{3}$ perovskite nanosheets.

3.3. Experimental Method. (1) X-Ray Diffraction Analysis. The X-ray diffraction test method is based on the diffraction effect of a polycrystalline sample on X-ray and analyzes and determines the presence of each component in the sample. $\mathrm{X}$-ray powder diffraction can not only analyze the phase of the sample, but also determine the unit cell parameters of the crystal structure, the lattice pattern, and the atomic coordinates of simple structures.

(2) Scanning Electron Microscope Morphology Analysis. Scan the different positions on the sample by scanning electron beams, collect this information, and send it to the imaging system after being magnified. The information emitted at any point in the sample surface scanning process can be recorded to obtain image information. The distribution of the surface morphology of the sample can be obtained through the correspondence between the 


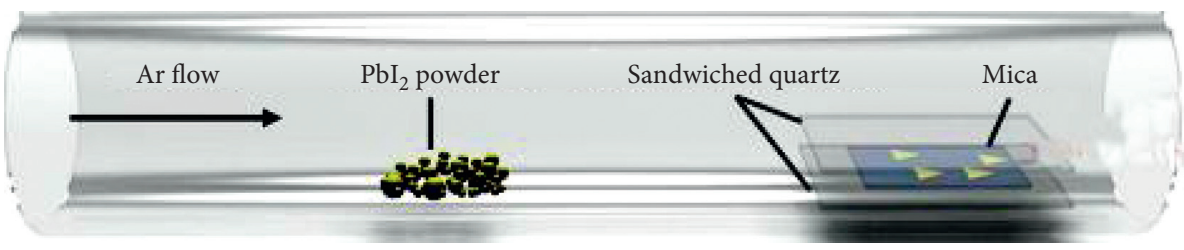

Figure 1: Schematic diagram of $\mathrm{PbI}_{2}$ nanosheets prepared by vapor deposition method (https://image.baidu.com/).

information and the sample position. First glue the conductive glue on the sample stage, then put an appropriate amount of sample on the conductive glue, use the blowing rubber ball to blow gently to blow off the excess sample, and make the sample evenly distributed on the conductive glue; finally, the sample stage is put into the gold spraying equipment, and the sample is sprayed gold and then taken out, to be tested.

(3) Analysis of the Morphology of the Transmission Electron Microscope. The electrons transmitted out of the sample are magnified and projected on the phosphor screen for observing the graph by the three-stage magnetic lens of the objective lens, the intermediate mirror, and the projection lens. Therefore, the image corresponding to the morphology, organization, and structure of the sample is displayed on the fluorescent screen.

\subsection{Establishment of the Model Evaluation Index System.} The evaluation index is a specific evaluation item that is determined according to the evaluation purpose and can reflect the basic characteristics of the evaluation target. Indicators are specific and measurable and are the observation points of your target. Clear conclusions can be drawn from the actual observation of the object. Typically, the metrics system includes three levels of metrics. This is the relationship between progressive decomposition and improvement. Among these, the first-stage evaluation index and the second-stage evaluation index are relatively abstract and cannot be used as direct evaluation criteria. The threelevel assessment indicator should be specific, measurable, and action-oriented and can be used as a direct basis for assessment.

3.5. Statistical Processing. Statistical analysis was performed with SPSS 13.0 statistical software, the significance test of the difference was performed by one-way analysis of variance, the difference between the two groups was tested by LSD- $t$, and the photoelectric properties of perovskite nanomaterials were performed by group $t$-test. $P<0.05$ is considered to be statistically significant.

\section{Construction and Photoelectric Properties of Perovskite Nanomaterials}

4.1. Evaluation Index System Based on Index Reliability Testing. Here we perform reliability analysis on all reliability indicators of each object. The reliability indicators we choose for each object are slightly different. The results are shown in Table 1 .
It can be seen from Figure 2 that the morphology control of the $\mathrm{PbI}_{2}$ nanosheets, the growth mechanism of $\mathrm{PbI}_{2}$, the characterization of the composition, and the data obtained from the optical properties have a very good effect on this experiment $(\alpha>0.8)$; the influence of the data obtained from various indicators of the optical performance of MAPbI3 nanosheets on this experiment is acceptable $(\alpha>0.7)$. This shows that the indicators are reasonable when studying the composition and photoelectric properties of the five selected chiral perovskite nanomaterials in this paper. It provides the basis for subsequent experiments.

4.2. Evaluation Index System Based on Inspection Data. (1) $\mathrm{PbI}_{2}$ Nanosheet Morphology Control. Keeping the growth temperature and substrate unchanged, the size of the confinement space and the distance between the substrate and the furnace center are important factors affecting the growth of $\mathrm{PbI}_{2}$ nanosheets. Therefore, the size of the confinement space (the height between the sandwich quartz plates) is compared here. The results of the experiment are shown in Figure 3.

Figure 3 shows the synthesized $\mathrm{PbI}_{2}$ nanosheets synthesized under different confinement space heights. The confinement space heights are $1 \mathrm{~mm}, 2 \mathrm{~mm}$, and $3 \mathrm{~mm}$, respectively. The crystal size and nucleation density of these samples were counted, and the results are shown in Table 2.

It can be seen from Figure 4 that as the height of the confined space increases from $1 \mathrm{~mm}$ to $3 \mathrm{~mm}$, the average crystal size increases from $4 \mu \mathrm{m}$ to $8 \mu \mathrm{m}$. But when the height of the confined space is $3 \mathrm{~mm}$, the synthesized $\mathrm{PbI}_{2}$ nanosheets are no longer uniformly distributed: many small particles and linear structures appear on the substrate. This is similar to the sample obtained without the confined space, indicating that as the height of the confined space increases, the confinement effect decreases. However, $\mathrm{PbI}_{2}$ nanosheets with uniform shape and crystal size distribution can be obtained at both $1 \mathrm{~mm}$ and $2 \mathrm{~mm}$ limit heights. The nucleation density gradually increases as the height of the confinement space increases, from $36.14 * 10^{4} \mathrm{~cm}^{2}$ at $1 \mathrm{~mm}$ to $125.48 * 10^{4} \mathrm{~cm}^{2}$ at $3 \mathrm{~mm}$, indicating that the size of the confinement space is positively correlated with the nucleation density. When the height of the confinement space increases to $3 \mathrm{~mm}$, the uniformity of the $\mathrm{PbI}_{2}$ nanosheets is reduced, and the confinement effect is weakened. Therefore, the effect is best when the height of the confined space is $2 \mathrm{~mm}$, and a uniform and relatively large $\mathrm{PbI}_{2}$ triangular nanosheet can be obtained.

(2) $\mathrm{PbI}_{2}$ Growth Mechanism. It can be seen from Figure 5 that for the case of no confined space, $\mathrm{PbI}_{2}$ has both 
TABLE 1: Data sheet of evaluation index system for index reliability testing.

\begin{tabular}{|c|c|c|c|c|c|c|}
\hline & Very clear & Clear & General & Not clear & Chaotic & Alpha \\
\hline $\mathrm{PbI}_{2}$ nanosheet morphology control & 3.98 & 3.81 & 4.51 & 0.79 & 0.47 & 0.7281 \\
\hline $\mathrm{PbI}_{2}$ growth mechanism & 3.55 & 3.65 & 4.45 & 0.47 & 0.54 & 0.7349 \\
\hline Component structure characterization & 3.87 & 3.86 & 4.76 & 0.66 & 0.49 & 0.7425 \\
\hline Optical properties & 3.58 & 4.14 & 4.70 & 0.52 & 0.41 & 0.7762 \\
\hline Optical properties of $\mathrm{MAPbI}_{3}$ nanosheets & 3.62 & 3.98 & 4.52 & 0.53 & 0.42 & 0.8294 \\
\hline
\end{tabular}

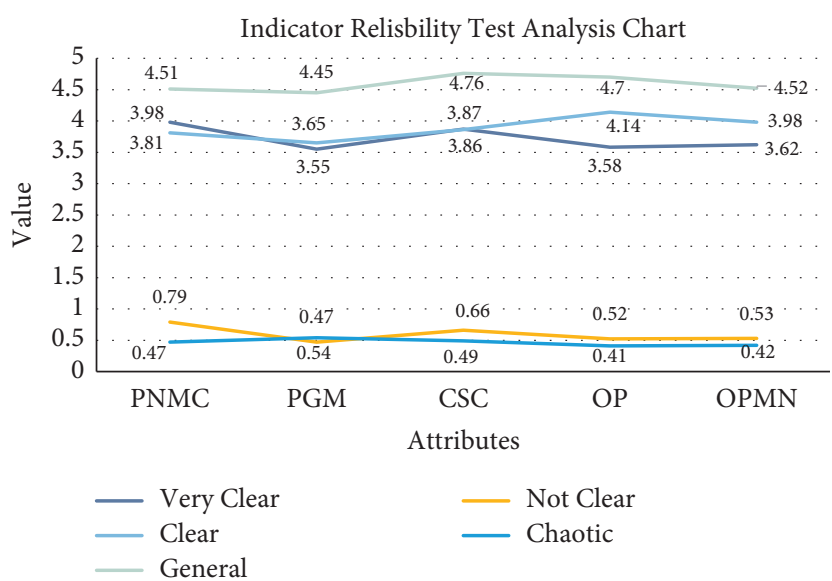

FIgURE 2: Indicator reliability test analysis chart.

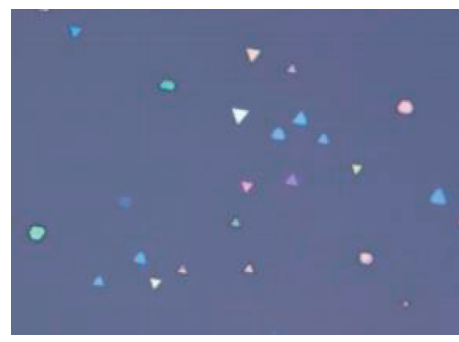

(a)

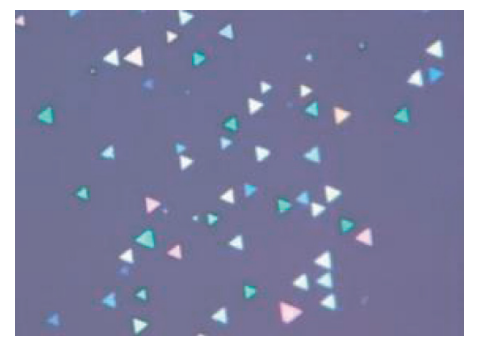

(b)

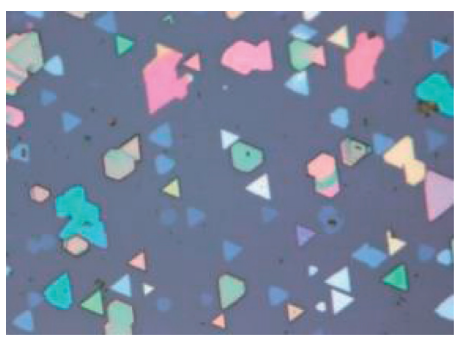

(c)

Figure 3: Influence of the height of the confined space on the morphology of $\mathrm{PbI}_{2}$ nanosheets (https://image.baidu.com/). (a) $\mathrm{PbI}$ nanosheets under the restricted space height of $1 \mathrm{~mm}$. (b) $\mathrm{PbI}_{2}$ nanosheets under the restricted space height of $2 \mathrm{~mm}$. (c) $\mathrm{PbI}_{2}$ nanosheets under the restricted space height of $3 \mathrm{~mm}$.

TABLE 2: Data sheet of evaluation index system for index reliability testing.

\begin{tabular}{lcc}
\hline Restricted space height & Average crystal size $(\mu \mathrm{m})$ & Nuclear density $\left({ }^{*} 10^{4} \mathrm{~cm}^{2}\right)$ \\
\hline $1 \mathrm{~mm}$ & 4 & 36.14 \\
$2 \mathrm{~mm}$ & 6 & 67.78 \\
$3 \mathrm{~mm}$ & 8 & 125.48 \\
\hline
\end{tabular}

triangular and hexagonal morphologies, indicating that the growth process has dynamic instability, and the concentration and flow rate of the gaseous source of $\mathrm{PbI}_{2}$ are constantly changing. This greatly limits the controllable growth of $\mathrm{PbI}_{2}$, thus limiting their further applications. When the sandwich quartz plate is introduced as the confined space, it cannot only ensure that the $\mathrm{PbI}_{2}$ gaseous source can be successfully transferred to the substrate, but also produce a stable gaseous source concentration delivery process, so that the gas source concentration is evenly distributed on the substrate. In the nucleation stage, the low source concentration in the confined space effectively reduces the nucleation density; in the epitaxial growth stage, it can provide a stable source supply and realize the uniform growth of $\mathrm{PbI}_{2}$ nanosheets. As the substrate distance increases, the $\mathrm{PbI}_{2}$ concentration gradually decreases, resulting in thinner nanosheets. The confined space reduces nucleation density and provides a relatively stable gas source 


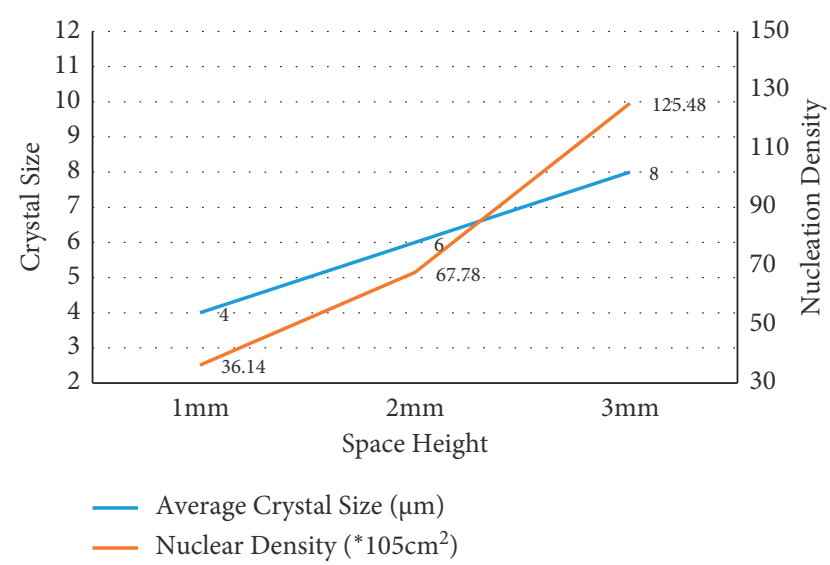

FIGURE 4: Relationship between crystal size and nucleation density and confinement space height.

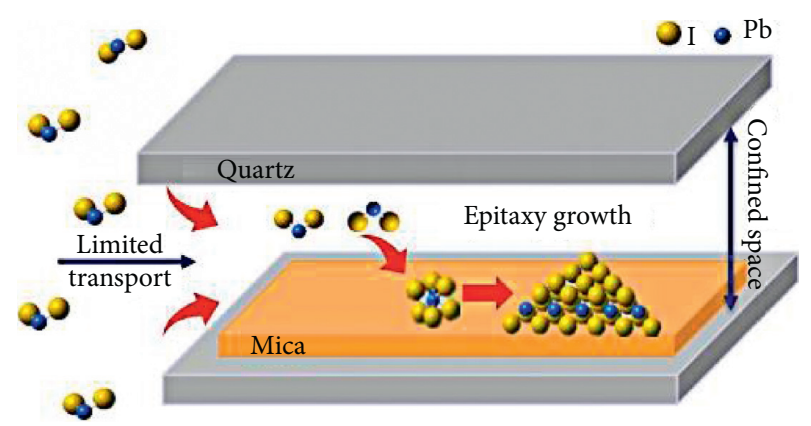

Figure 5: Schematic diagram of the growth mechanism of $\mathrm{PbI}_{2}$ nanosheets in a confined space (https://image.baidu.com/).

supply environment, which can overcome dynamic instability during $\mathrm{PbI}_{2}$ growth and achieve uniform and controllable preparation for the shape and thickness of $\mathrm{PbI}_{2}$ nanosheets.

(3) Characterization of Composition Structure. Through $\mathrm{X}$-ray diffraction pattern, $\mathrm{X}$-ray photoelectron spectroscopy, the phase structure, and chemical state of $\mathrm{PbI}_{2}$ nanosheets were studied. The X-ray diffraction pattern was used to determine the crystal phase structure of the sample, and the results are shown in Table 3.

It can be seen from Figure 6 that all the peak positions in the figure are in good agreement with the hexagonal phase $\mathrm{PbI}_{2}$, and only the (001) plane diffraction peak appears, which indicates that the out-of-plane growth direction of $\mathrm{PbI}_{2}$ nanosheets is the [001] direction.

We conducted transmission electron microscopy analysis of the $\mathrm{PbI}_{2}$ crystal structure, and the transmission electron microscopy sample was obtained by sample transfer. The result is shown in Figure 7.

The high-resolution electron microscope image of the $\mathrm{PbI}_{2}$ nanosheet is shown in Figure $7(\mathrm{a})$. The distance between the crystal planes is $0.228 \mathrm{~nm}$, which corresponds to the (110) plane of $\mathrm{PbI}_{2}$. The selected area electron diffraction pattern in Figure 7(b) also proves that the high-resolution exposed crystal plane is the $\mathrm{PbI}_{2}(110)$ crystal plane, and the direction perpendicular to the crystal plane is the [001] crystal orientation. The results obtained are also consistent
TABLE 3: Component structure characterization data sheet.

\begin{tabular}{lcccc}
\hline Degree & $(001)$ & $(002)$ & $(003)$ & $(004)$ \\
\hline 10 & 1.29 & 2.59 & 2.31 & 2.75 \\
20 & 2.68 & 2.70 & 2.80 & 3.39 \\
30 & 3.43 & 3.97 & 3.47 & 4.20 \\
40 & 3.45 & 4.24 & 3.76 & 4.78 \\
50 & 4.46 & 4.44 & 4.96 & 5.38 \\
60 & 4.32 & 5.02 & 4.94 & 5.51 \\
\hline
\end{tabular}

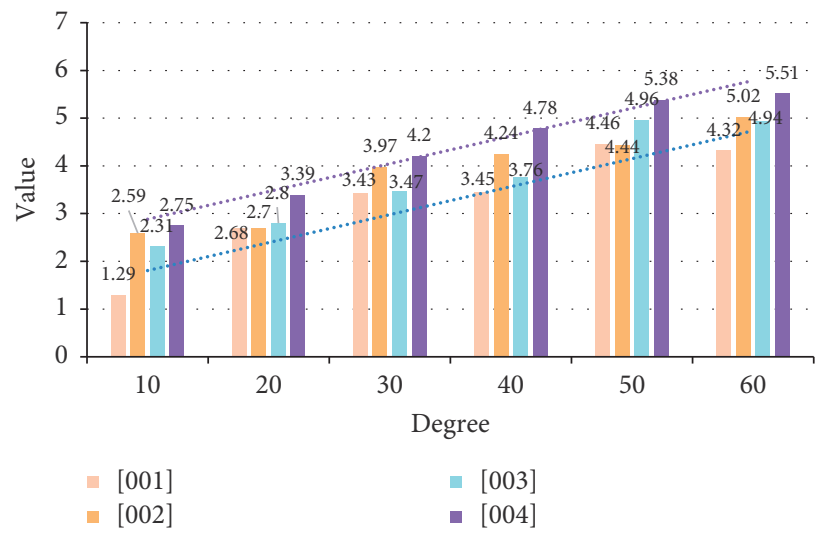

FIGURE 6: Component structure characterization analysis diagram.

with the X-ray diffraction pattern results. These all indicate that the obtained $\mathrm{PbI}_{2}$ nanosheets are single crystals with high quality and uniformity.

(4) Optical Property Analysis. The optical properties of $\mathrm{PbI}_{2}$ nanosheets were studied, and the Raman peak positions of samples with different thicknesses were studied. The results are shown in Table 4.

It can be seen from Figure 8 that the Raman peak of the mica substrate is relatively weak, and its influence on the test results can be ruled out. All samples have characteristic peaks at 75,100 , and 125 , which are attributed to the three vibration mode peaks of $E_{g}, A_{1 g}$, and $A_{2 u}$ in $\mathrm{PbI}_{2}$, respectively. As the thickness of $\mathrm{PbI}_{2}$ increases, the peak intensity of all peaks becomes stronger and the half-width becomes narrower, but the position of the characteristic peak remains unchanged.

(5) Study on Gas Phase Transformation and Optical Properties of $\mathrm{MAPbI}_{3} \mathrm{Nanosheets}$ Through precise control of reaction conditions and related characterization, we have obtained high-quality $\mathrm{PbI}_{2}$ nanosheets with uniform morphology and controllable size, which provides an important basis for the two-step preparation of $\mathrm{MAPbI}_{3}$ nanosheets. The obtained $\mathrm{MAPbI}_{3}$ nanosheets were characterized by related optical properties, and the results are shown in Table 5.

It can be seen from Figure 9 that the peak position of the fluorescence spectrum obtained by Gaussian fitting is $800 \mathrm{~nm}$, and the half-value width is about $45 \mathrm{~nm}$. The halfvalue width can reflect the crystalline properties of the material, and the smaller the value, the better, which fully indicates that the obtained nanosheets have high crystallinity and at the same time indicates that the converted perovskite 


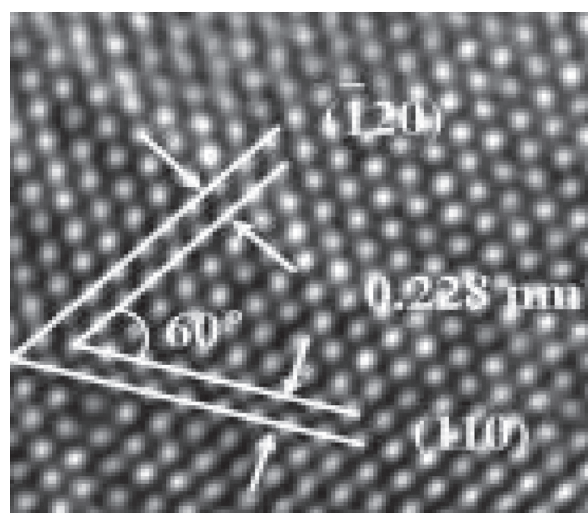

$\mathrm{PbI}_{2}$ high resolution transmission spectrum

(a)

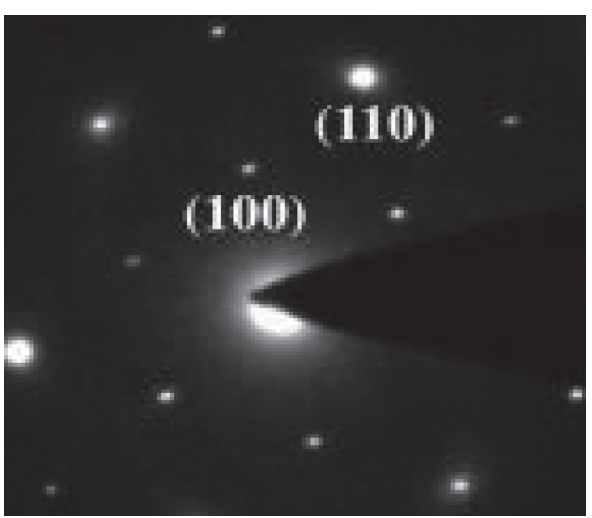

$\mathrm{PbI}_{2}$ high-resolution corresponding selected area electron diffraction pattern

(b)

FIgURE 7: Transmission electron microscope analysis chart (https://image.baidu.com/).

TABLE 4: Optical properties data sheet.

\begin{tabular}{lccccccc}
\hline Raman shift & Mica & $5 \mathrm{~nm}$ & $10 \mathrm{~nm}$ & $30 \mathrm{~nm}$ & $40 \mathrm{~nm}$ & $80 \mathrm{~nm}$ & 2.36 \\
\hline 50 & 2.58 & 2.46 & 2.98 & 2.34 & 2.98 & 2.83 & 3.43 \\
75 & 2.73 & 3.17 & 3.53 & 2.73 & 2.58 & 3.04 \\
100 & 2.57 & 2.97 & 2.77 & 2.76 & 3.58 & 2.45 & 3.51 \\
125 & 3.95 & 3.21 & 3.5 & 3.85 & 3.42 & 3.06 \\
150 & 2.79 & 2.71 & 2.86 & 2.07 & 2.79 \\
175 & 2.66 & 2.84 & 2.78 & 2.63 & 2.96 & 2.97 \\
\hline
\end{tabular}
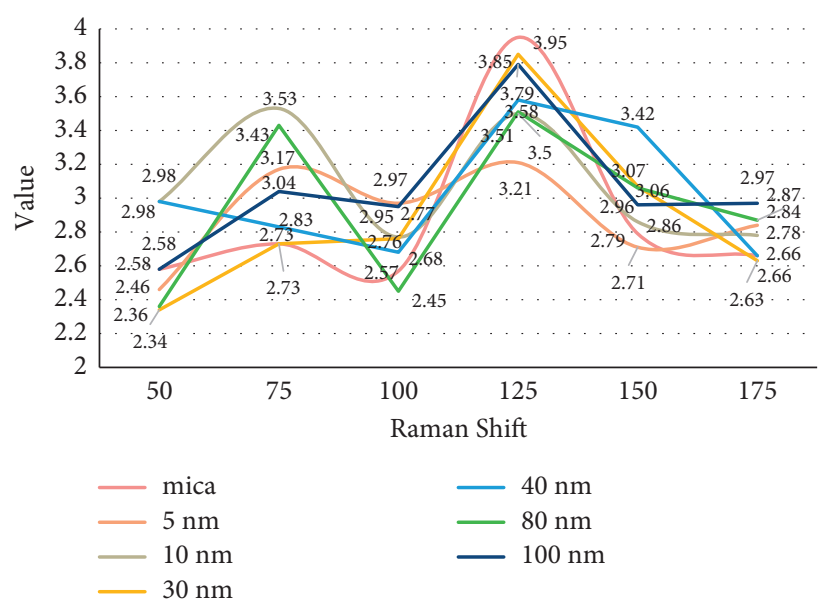

Figure 8: Optical property analysis chart.

TABLE 5: $\mathrm{MAPbI}_{3}$ nanosheet optical performance data sheet.

\begin{tabular}{lccc}
\hline Wavelength & MAPbI $_{3}$ & $\mathrm{PbI}_{2}$ & \\
\hline 550 & 0.1 & 0.3 & Mica \\
600 & 0.1 & 0.3 & 0.5 \\
650 & 0.1 & 0.3 & 0.5 \\
700 & 0.1 & 0.3 & 0.5 \\
750 & 0.4 & 0.6 & 0.5 \\
800 & 1.0 & 1.4 & 0.8 \\
850 & 0.4 & 0.6 & 2.0 \\
900 & 0.1 & 0.3 & 0.8 \\
950 & 0.1 & 0.3 & 0.5 \\
\hline
\end{tabular}




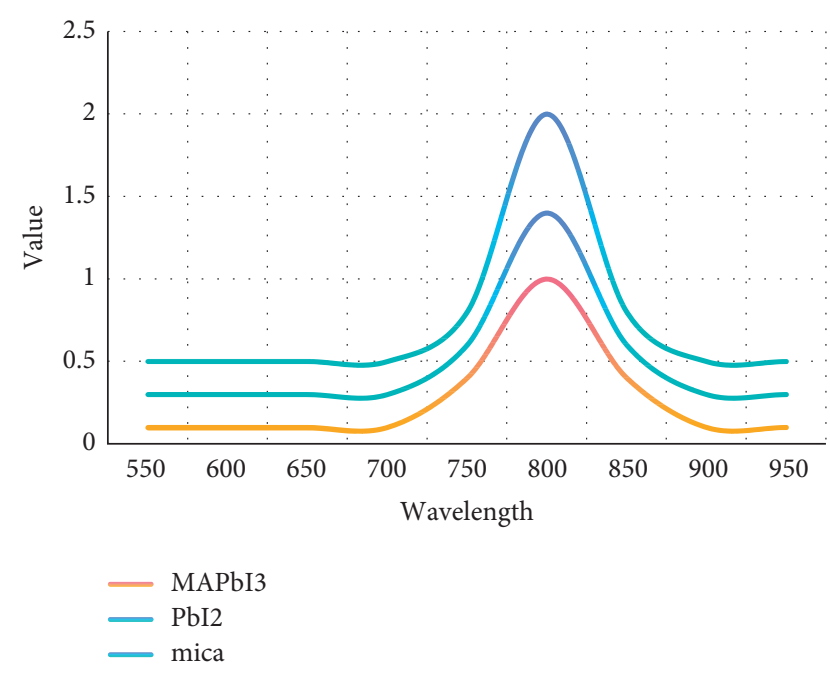

FIgURE 9: $\mathrm{MAPbI}_{3}$ nanosheet optical performance analysis diagram.

nanosheets have good uniformity. $\mathrm{MAPbI}_{3}$ exhibits strong light absorption characteristics when the wavelength is less than $800 \mathrm{~nm}$, which also proves that $\mathrm{MAPbI}_{3}$ not only has good fluorescence spectrum luminescence properties, but also has strong light absorption characteristics.

\section{Conclusions}

The gas phase method is an important method for preparing high-quality perovskite nanomaterials. Improving the controllability of the gas phase method and reducing the production cost are of great significance for further performance research and commercial application of the perovskite. This paper takes organic perovskite $\mathrm{MAPbI}_{3}$ nanosheets as the research object, through the improvement of the gas phase method and the introduction of limited space to improve the controllability of the preparation of precursor $\mathrm{PbI}_{2}$ nanosheets, then obtain uniform $\mathrm{MAPbI}_{3}$ nanosheets, and further explore its growth mechanism and study its photoelectric performance. By introducing a limited space, the $\mathrm{PbI}_{2}$ nanosheets with uniform and controllable morphology, size, and thickness were successfully prepared by physical vapor deposition. The confined space is the key to realize the uniform growth of $\mathrm{PbI}_{2}$ nanosheets. It provides a relatively stable growth environment and avoids the dynamic instability during the growth of $\mathrm{PbI}_{2}$. This confined space vapor deposition method can improve the uniformity and controllability of the preparation of $\mathrm{PbI}_{2}$ and $\mathrm{MAPbI}_{3}$ nanosheets, which provides a basis for further research of $\mathrm{MAPbI}_{3}$ nanomaterials and also has reference significance for the controllable growth of other nanomaterials.

The introduction of chirality into the optical communication system can not only increase another new dimension of high-speed optical information transmission, but also significantly increase the information capacity. Therefore, chiral perovskite nanomaterials provide broad application prospects in this regard. This paper mainly introduces a limited space to suppress the dynamic instability during the $\mathrm{PbI}_{2}$ vapor deposition process, so as to obtain high-quality $\mathrm{PbI}_{2}$ nanosheets with uniform and controllable morphology and thickness. At the same time, $\mathrm{PbI}_{2}$ can be converted into $\mathrm{MAPbI}_{3}$ nanosheets by chemical vapor deposition, which provides a method for the uniform and controllable synthesis of two-dimensional perovskite and also provides a basis for its performance research. The current field of chiral perovskites is a brand-new research field, which is both an opportunity and a challenge. A lot of basic and applied work needs to be carried out.

This paper improves the perovskite vapor phase synthesis method, reduces the cost of preparing the vapor phase method, and obtains a uniform and controllable perovskite nanomaterial. Although we have achieved some results from the preparation of the material, there are still aspects that require further development. Although uniform and controllable $\mathrm{PbI}_{2}$ and $\mathrm{MAPbI}_{3}$ nanosheets were obtained with a limited space method, in-depth studies on that method and the material obtained are lacking. Movement instability during growth of nanomaterials is universal. This experiment improves the preparation of $\mathrm{PbI}_{2}$ nanosheets and overcomes this phenomenon. However, the same problems that exist in the fabrication of other nanomaterials have not been explored in depth.

\section{Data Availability}

No data were used to support this study.

\section{Conflicts of Interest}

The author declares that there are no conflicts of interest regarding the publication of this article.

\section{References}

[1] S. T. Ha, R. Su, J. Xing, Q. Zhang, and Q. Xiong, "Metal halide perovskite nanomaterials: synthesis and applications," Chemical Science, vol. 8, no. 4, pp. 2522-2536, 2017.

[2] B. Halford, "Nanomaterials Chiral gold nanoparticles," Chemical and Engineering News: |"news Edition।" of the American Chemical Society, vol. 96, no. 17, pp. 10-11, 2018.

[3] M. M. Riba, C. G. Oliveras, D. B. Amabilino, and A. C. González, "Supramolecular block copolymers incorporating chiral and achiral chromophores for the bottom-up assembly of nanomaterials," Journal of Porphyrins \& Phthalocyanines, vol. 23, no. 07n08, pp. 916-929, 2019.

[4] X. Shang, I. Song, H. Ohtsu et al., "Supramolecular nanostructures of chiral perylene diimides with amplified chirality for high-performance chiroptical sensing," Advanced Materials, vol. 29, no. 21, pp. 1605828.1-1605828.7, 2017.

[5] B. Han, L. Shi, X. Gao et al., "Ultra-stable silica-coated chiral Au-nanorod assemblies: core-shell nanostructures with enhanced chiroptical properties," Nano Research, vol. 9, no. 2, pp. 451-457, 2016.

[6] H. Kong, X. Sun, L. Yang, X. Liu, H. Yang, and R. H. Jin, "Chirality detection by Raman spectroscopy: the case of enantioselective interactions between amino acids and polymer-modified chiral silica," Analytical Chemistry, vol. 92, no. 21, pp. 14292-14296, 2020. 
[7] S. Wang, J. Yu, J. Li, and Y. Lin, "Prevalence and imaging features of superior canal dehiscence based on HRCT," Chinese Journal of Medical Imaging Technology, vol. 34, no. 10, pp. 1465-1468, 2018.

[8] V. Y. Gus'kov and V. N. Maistrenko, "New chiral stationary phases: preparation, properties, and applications in gas chromatography," Journal of Analytical Chemistry, vol. 73, no. 10, pp. 937-945, 2018.

[9] X. Wang, B. Liu, Y. Yu et al., "Application value of chest multi-detector spiral CT in diagnosis and follow-up of corona virus disease 2019," Chinese Journal of Medical Imaging Technology, vol. 36, no. 3, pp. 400-404, 2020.

[10] M. C. Weidman, A. J. Goodman, and W. A. Tisdale, "Colloidal halide perovskite nanoplatelets: an exciting new class of semiconductor nanomaterials," Chemistry of Materials: A Publication of the American Chemistry Society, vol. 29, no. 12, pp. 5019-5030, 2017.

[11] M. Jiang, W. Guo, T. Jiao, and Q. Xu, "Metal ion substitution effect and component regulation of perovskite-type $\mathrm{La}_{1-\mathrm{x}} \mathrm{Ca}_{\mathrm{x}}$ $\mathrm{CrO}_{3}$ nanomaterials," Science of Advanced Materials, vol. 9, no. 7, pp. 1231-1235, 2017.

[12] N. A. Gibson, B. A. Koscher, A. P. Alivisatos, and S. R. Leone, "Excitation intensity dependence of photoluminescence blinking in $\mathrm{CsPbBr} 3$ perovskite nanocrystals," The Journal of Physical Chemistry, C. Nanomaterials and Interfaces, vol. 122, no. 22, pp. 12106-12113, 2018.

[13] A. S. Alsubaie, "Recent advances in bismuth ferrite nanomaterials: synthesis, characterization and application," Journal of Chemical, Biological and Physical Sciences, vol. 11, no. 2, pp. 101-111, 2021.

[14] M. Yamauchi, Y. Fujiwara, and S. Masuo, "Slow anion-exchange reaction of cesium lead halide perovskite nanocrystals in supramolecular gel networks," ACS Omega, vol. 5, no. 24, pp. 14370-14375, 2020.

[15] H. Wang, Y. Liang, L. Liu, J. Hu, P. Wu, and W. Cui, "Enriched photoelectrocatalytic degradation and photoelectric performance of BiOI photoelectrode by coupling rGO," Applied Catalysis B Environmental, vol. 208, pp. 22-34, 2017.

[16] B. Hu, Y. Wang, C. Hu, and X. F. Zhou, "Design, fabrication and high efficient visible-light assisted photoelectric-synergistic performance of 3-D mesoporous DSA electrodes," Materials and Design, vol. 91, pp. 201-210, 2016.

[17] Y. Jun, Y. Li, Z. Jia, and Z. Dong, "Enhanced photoelectric performance of composite nanostructures combining monolayer graphene and a RbAg4I5 film," Applied Physics Letters, vol. 110, no. 21, pp. 1-4, 2017.

[18] W. Luan, C. Zhang, L. Luo, B. Yuan, L. Jin, and Y. S. Kim, "Enhancement of the photoelectric performance in inverted bulk heterojunction solid solar cell with inorganic nanocrystals," Applied Energy, vol. 185, no. 2, pp. 2217-2223, 2016.

[19] H. Li and W. Gao, "Detection sensitivity calculation model and photoelectric detection performance analysis on laser light screens," IEEE Sensors Journal, vol. 16, no. 11, pp. 4258-4264, 2016.

[20] Y. Deng, Z. Ma, F. Ren, and G. Wang, "Improved photoelectric performance of DSSCs based on TiO2 nanorod array/ Ni-doped TiO2 compact layer composites film," Journal of Solid State Electrochemistry, vol. 23, no. 11, pp. 3031-3041, 2019.

[21] F. Li, G. Chen, and X. Fu, "Erratum to: comparison of effect of gear juicer and colloid mill on microstructure, polyphenols profile, and bioactivities of mulberry (morus indica L.)," Food and Bioprocess Technology, vol. 9, no. 7, pp. 1246-1248, 2016.
[22] Y. S. Shmaliy, S. H. Khan, S. Zhao, and O. M. Ibarra, "General unbiased FIR filter with applications to GPS-based steering of oscillator frequency," IEEE Transactions on Control Systems Technology, vol. 25, no. 3, pp. 1141-1148, 2017.

[23] N. Rozzani, I. S. Mohamed, and S. A. N. S. Yusuf, "Technology for Islamic microfinance's disbursement and repayment system," International Journal of Social Economics, vol. 43, no. 12, pp. 1271-1283, 2016.

[24] A. Abburi, M. Ali, and P. V. Moriya, "Synthesis of mesoporous silica nanoparticles from waste hexafluorosilicic acid of fertilizer industry," Journal of Materials Research and Technology, vol. 9, no. 4, pp. 8074-8080, 2020.

[25] K. Reals, "Industry urges improved access to aircraft cabins for the disabled," Aviation Week \& Space Technology, vol. 180, no. 13, pp. 70-71, 2018. 